\title{
Femtosecond single-shot timing and direct observation of subpulse formation in an infrared free-electron laser
}

\author{
R. Kiessling, ${ }^{1, \dagger}$ W. B. Colson, ${ }^{2}$ S. Gewinner, ${ }^{1}$ W. Schöllkopf, ${ }^{1}$ M. Wolf, ${ }^{1}$ and A. Paarmann ${ }^{1, *}$ \\ ${ }^{1}$ Fritz Haber Institute of the Max Planck Society, Faradayweg 4-6, 14195 Berlin, Germany \\ ${ }^{2}$ Compass Scientific Engineering, Fremont, California 94539, USA
}

(Received 3 April 2018; published 20 August 2018)

\begin{abstract}
We experimentally demonstrate a single-shot arrival time monitor for short picosecond infrared freeelectron laser (IR FEL) pulses based on balanced optical cross-correlation with a synchronized fs table-top laser. Employing this timing tool at the Fritz Haber Institute IR FEL, we observe a shot-to-shot timing jitter of only 100 fs and minute-scale timing drifts of a few picoseconds, the latter being strictly correlated with the electron beam energy of the accelerator. We acquire sum-frequency cross-correlation data with micropulse resolution, providing full access to the IR FEL pulse shape evolution within the macropulse. These measurements provide unprecedented insights into the occurrence of limit-cycle oscillations of the FEL intensity as a consequence of subpulse formation. Our experimental results are complemented by fourdimensional simulations of the nonlinear pulse dynamics in a low-gain FEL oscillator based on MaxwellLorentz theory.
\end{abstract}

DOI: 10.1103/PhysRevAccelBeams.21.080702

\section{INTRODUCTION}

The availability of extremely intense, spectrally tunable coherent light pulses facilitates progress in various scientific fields due to the possibility to perform optical spectroscopy [1] and imaging [2,3] of matter on its natural time and length scales. The concept of the free-electron laser allows to generate ultrafast optical pulses from the $\mathrm{THz}$ to the hard $\mathrm{x}$-ray regime, determined solely by the electron bunch length and energy. Thus, ultrashort relativistic electron pulses have to be employed which, for x-ray FELs, are typically of femtosecond duration [4].

Infrared FEL oscillators, on the other hand, are usually operated using picosecond-long electron pulses [5,6]. Here, significant short pulse effects in the FEL interaction can occur on the sub-ps scale [5,7]. Due to the large slippage length as compared to the electron pulse duration, the lasing dynamics are quite sensitive to the temporal overlap of the electron and optical pulses. Since in an FEL oscillator the length of the resonator is a critical degree of freedom to control this overlap, a manifold of pulse formation regimes are possible. Thus, proper characterization and understanding of the optical FEL output across the different regimes of

\footnotetext{
*Corresponding author. alexander.paarmann@fhi-berlin.mpg.de

'kiessling@fhi-berlin.mpg.de

Published by the American Physical Society under the terms of the Creative Commons Attribution 4.0 International license. Further distribution of this work must maintain attribution to the author(s) and the published article's title, journal citation, and DOI.
}

operation is a prerequisite for successful application in experiments making use of the short FEL pulses in timeresolved and multicolor spectroscopic approaches. For experimental studies combining FEL and table-top laser sources, the exact pulse arrival time is of utmost importance, besides the intrinsic FEL pulse properties like polarization, temporal or spectral shape and pulse energy. In order to take full advantage of the ultrashort FEL pulses to study the dynamics of matter with high time resolution, precise synchronization and/or timing monitoring is required.

So far, different methods have been engaged to capture FEL pulse-to-pulse variations of the arrival time due to electron bunch charge fluctuations and linear accelerator noise with respect to the phase-locked external laser. A rather indirect approach is to measure the timing of the electron bunches that generate the optical pulse by electro-optic sampling of the electric field that accompanies the ultrarelativistic particles [8]. However, direct cross-correlation of the table-top light with the FEL radiation has been applied in the IR regime (by sum-frequency generation $[9,10]$ ) and recently also to fs X-ray pulses (e.g., via induced transient changes of the optical reflectivity $[11,12])$.

Here, we extend these concepts of pulse arrival determination at IR FELs by utilizing balanced optical crosscorrelation (BOC) to improve the time resolution. This method, applied so far to optical lasers [13,14], allows access to the absolute delay between accelerator-based and table-top light pulses with fs-accuracy. In this paper, we demonstrate the BOC concept employing a fs table-top near-IR laser synchronized with an IR FEL at the Fritz Haber Institute (FHI) using a low-jitter radio-frequency phaselocking system. The balanced cross-correlation signal is 
provided by sum-frequency generation in an appropriate nonlinear medium. Monitoring of the temporal pulse overlap parallel to the actual experiment allows for post-correction and therefore, fluctuations in time-resolved studies can be eliminated $[12,15]$.

Due to the high level of synchronization, the crosscorrelation function provides, on the other hand, a direct measurement of the FEL pulse intensity profile and its evolution over time. This enables a detailed study of the FEL process itself. FEL theory has now been developed so far as to quantitatively predict the length and shape of the generated optical pulses. A classical treatment of the relativistic electrodynamics based on Maxwell-Lorentz equations emerged to be a valid description of the energy exchange between particles and optical field [16,17]. Whereas the underlying physical process for the optical power growth is already captured by a $1 \mathrm{D}$ analysis, the study of the pulse profile requires 2D simulations [18]. The most complete simulations use the self-consistent Maxwell-Lorentz equations in four dimensions $(x, y, z, t)$ including a long list of relevant effects like electron beam emittance, betatron motion, energy spread, arbitrary optical mode and pulse shape evolution. The 4D simulation used in this paper has been studied extensively and compared to many FEL amplifier and oscillator experiments [19]. Furthermore, pronounced nonlinear processes like subpulse formation and limit-cycle oscillations (LCOs) of the power have been predicted for short electron bunches within an oscillator-type FEL [20,21]. Our experimental cross-correlation measurements provide both delay-time as well as real-time resolution and thus give insight into these processes in yet unprecedented contrast and detail, as opposed to previous one-dimensional studies [5,7]. The results are complemented by $4 \mathrm{D}$ simulations of the FEL process, which confirm the pulse shape evolution and guide the understanding of the FEL oscillator physics.

The paper is organized as follows: First, we describe the experimental system consisting of the IR FEL, the table-top laser and synchronization. Then, the BOC method is introduced and applied to characterize the FEL pulse timing stability, i.e., jitter and drift. Subsequently, cavity detuningdependent pulse profile measurements by cross-correlation of the FEL output with the table-top pulse are presented. Specifically, we analyze the evolution of the IR temporal pulse shape as a function of the number of resonator roundtrips, observing subpulse growth and LCOs of the power envelope. Finally, the experimental results are compared to numerical simulations of the FEL oscillator based on Maxwell-Lorentz theory, showing qualitative agreement.

\section{EXPERIMENTAL SYSTEM}

\section{A. FHI free-electron laser}

The free-electron laser installed at the FHI is an oscillatortype source of coherent IR radiation, currently operating in the spectral range from 3 to $50 \mu \mathrm{m}$. In the following, a brief description of the system is given. Further technical details are described in Ref. [6]. The electron bunches emitted from a thermionic cathode are first compressed in time by a buncher cavity and then accelerated by two subsequent normal-conducting linacs, operating at the frequency $f_{\mathrm{MO}}=$ $2.99 \mathrm{GHz}$ of the master oscillator (MO). The MO itself consists of a quartz oscillator clock operating at $100 \mathrm{MHz}$, which is electronically converted to $f_{\mathrm{MO}}$. The repetition rate of the gridded electron gun is set to the third sub-harmonic of the rf $\mathrm{MO}(1.0 \mathrm{GHz})$ in the standard mode of operation, or to the 18th or 36th fraction of this, i.e., $55.5 \mathrm{MHz}$ or $27.8 \mathrm{MHz}$, in the reduced repetition rate mode. Electron microbunches of $>200 \mathrm{pC}$ charge and few picosecond duration are obtained with a kinetic energy between 15 and $50 \mathrm{MeV}$, depending on the desired IR spectral range, and less than $0.3 \%$ energy spread. A train of electron microbunches with usually $\sim 10 \mu$ s duration per shot is repeated at a rate of $10 \mathrm{~Hz}$, delivering short ps optical FEL micropulses bundled into macropulses.

The planar hybrid-magnet $(\mathrm{NdFeB})$ undulator contains $N=50$ periods of $\lambda_{\mathrm{U}}=40 \mathrm{~mm}$ length. Scanning the gap between the magnet banks allows adjusting the undulator strength parameter from $K=0.5$ to $1.6(\mathrm{rms})$. The FEL cavity of $L_{0}=5.4 \mathrm{~m}$ nominal length is formed by goldplated concave spherical mirrors, whereas light out-coupling on one end is achieved by a $2.5 \mathrm{~mm}$ diameter hole. An additional degree of freedom is provided by the cavity detuning $\Delta L$, resulting in a shortening of the cavity length to $L_{0}-\Delta L$. Given the resonator round-trip time of $36 \mathrm{~ns}$, the reduced electron microbunch rate of $27.8 \mathrm{MHz}(55.5 \mathrm{MHz})$ corresponds to just one (two) optical micropulse circulating the FEL cavity. In the standard $1 \mathrm{GHz}-$ mode of operation, on the contrary, 36 optical pulses are circulating the FEL resonator simultaneously.

The center wavelength of the emitted FEL radiation is determined by the resonance condition, $\lambda=\lambda_{\mathrm{U}}\left(1+K^{2}\right) /$ $2 \gamma^{2}$, where $\gamma$ is the Lorentz factor. The spectrum is monitored for each optical macropulse by a CzernyTurner grating spectrometer combined with a pyroelectric array detector. Due to the planar undulator design, the IR light is linearly polarized [16], as confirmed by our measurements [6]. Intrinsically generated higher-order harmonics of the midinfrared (MIR) wavelength $\lambda$ have also been observed in the FHI FEL output, up to the 11th order [22]. To avoid absorption in ambient air, the FEL radiation is transported in an evacuated beamline, passing through a window made of KRS-5 to the user station.

\section{B. Table-top laser and synchronization}

The table-top laser employed is a high-power Terbiumdoped fiber oscillator (FO), providing bandwidth-limited, near-infrared (NIR, $\lambda=1055 \mathrm{~nm}$ ) soliton-shaped pulses of $\sim 100$ fs duration with up to $50 \mathrm{~nJ}$ energy [23]. The repetition rate of $f_{\mathrm{FO}}=55.5 \mathrm{MHz}$ is matched to the reduced electron 
microbunch rate at the FEL. Free-running timing jitter of the low phase-noise FO is specified to $<50$ fs rms [1 kHz$10 \mathrm{MHz}$. For high-precision electronic synchronization, the rf MO signal of $2.99 \mathrm{GHz}$ is distributed from the FEL vault to the user station via a commercial timing-stabilized rf-over-fiber link [24] approximately $100 \mathrm{~m}$ long. This reference clock transfer system is a low-jitter $(<7 \mathrm{fs}$ rms $[10 \mathrm{~Hz}-10 \mathrm{MHz}])$ and low-drift (<40 fs/day) rf transmission device utilizing two optical fiber connections to compensate phase drifts of the fiber cable due to environmental fluctuations [25]. The FO is phase-locked to the rf MO using the 54th harmonic of the table-top laser output received by a fast photodiode. The bandwidth of the custombuild phase-locked loop (PLL) is limited to $>1 \mathrm{kHz}$. Coarse adjustment of the FO repetition rate is achieved by temperature control of the laser cage (bandwidth $2.5 \mathrm{kHz}$ ), whereas fine tuning of the FO cavity length is realized by a piezoelectric transducer (capture range $>250 \mathrm{~Hz}$ ).

To accomplish temporal overlap of the fiber oscillator pulses at rate $f_{\mathrm{FO}}$ and FEL pulses using the higher frequency reference clock $f_{\mathrm{MO}}$, a phase-shifter of the $2.99 \mathrm{GHz}$ signal is implemented. Based on an rf vector-modulator [26], it allows a timing delay from 80 fs up to several tens of nanoseconds. For superperiod synchronization, the FO repetition rate is compared to a $55.5 \mathrm{MHz}$ rf signal derived from the MO at the FEL facility, which is transferred via a separate, non-stabilized fiber link [27]. Coarse temporal overlap of the NIR FO and MIR FEL pulses is determined by a fast $(\tau<1 \mathrm{~ns})$ IR-active photoelectromagnetic ( $\mathrm{HgCd}) \mathrm{Te}$-based detector [28], operational in a wavelength range from $500 \mathrm{~nm}$ to above $15 \mu \mathrm{m}$, in connection with a $4 \mathrm{GHz}$ oscilloscope.

\section{FEL PULSE TIMING}

\section{A. Principle of balanced optical cross-correlation}

In order to characterize the timing stability of the synchronized FO pulses with respect to the FEL micropulses, a balanced optical cross-correlation method has been employed [14]. See Fig. 1. In general, the relative timing between the pulse trains of two lasers can be directly measured by an optical cross-correlator. In order to extract an absolute timing, the cross-correlation is done twice to balance the signals, such that the timing value is directly proportional to the resulting difference signal. This is accomplished by inserting a small, but fixed time delay $\Delta t$ between the two pulse trains before the second crosscorrelation signal is generated [Fig. 1(a)]. Here, we choose sum-frequency generation (SFG) in a nonlinear crystal to acquire the cross-correlation function. After normalization and subtraction of both sum-frequency signals, the resulting BOC value is, within a given time delay window, proportional to the absolute delay $\tau$, characterized by a calibration coefficient $c$ :

$\operatorname{BOC}(\tau, \Delta t)=\frac{\mathrm{XC}(\tau)}{\max [\mathrm{XC}(\tau)]}-\frac{\mathrm{XC}(\tau+\Delta t)}{\max [\mathrm{XC}(\tau+\Delta t)]}=c \tau$.

The cross-correlation as well as BOC functions are illustrated in Fig. 1(b). For a properly chosen fixed delay $\Delta t$, depending on the duration of the input pulses, the dynamic range, in which the $\mathrm{BOC}$ signal is proportional to the pulse timing around $\tau=0$, is maximized. First demonstrated as an active feedback technique in all-optical table-top laser synchronization [13], the two-color BOC method allows us to characterize the timing stability of the phase-locked FEL-table-top laser system.

For calibration, the full BOC curve is acquired by scanning the time delay $\tau$ of the FO pulse with respect to the FEL micropulse via a motorized translation stage for fixed $\Delta t$. The coefficient $c$ is determined from the slope of a linear fit near $\tau=0$. To monitor the real-time development, the stage is set to a constant position within the linear range, and the measured BOC value is converted to the timing information $\tau$ using the calibration coefficient.

\section{B. Optical setup}

For the cross-correlation measurement we have chosen nonresonant bulk sum-frequency generation of MIR FEL
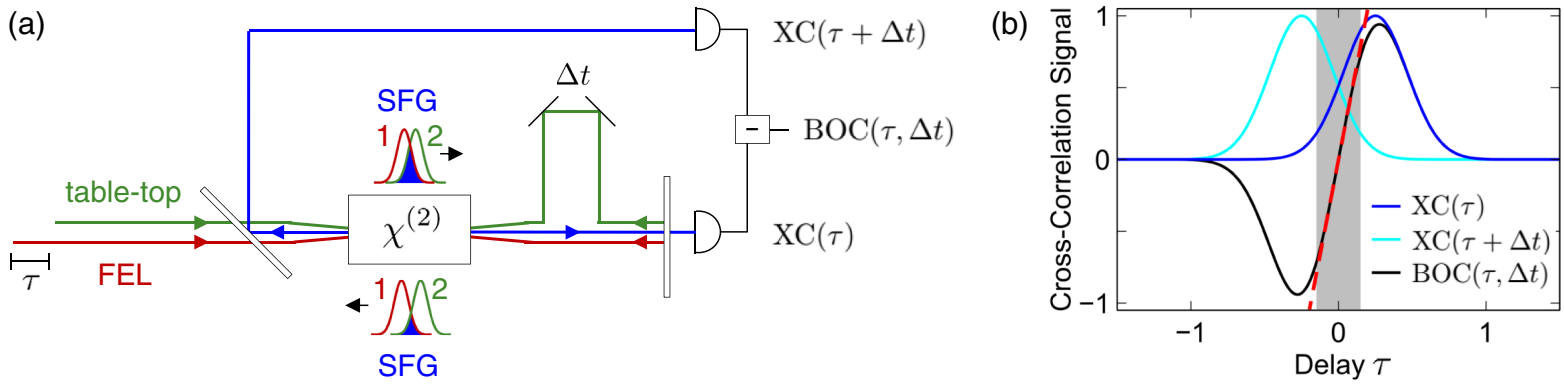

FIG. 1. Principle of two-color balanced optical cross-correlation. (a) Experimental scheme. Sum-frequency generation of FEL and table-top laser radiation is done twice within a single nonlinear crystal $\left(\chi^{(2)} \neq 0\right)$ after inserting a small, fixed time delay $\Delta t$ between FEL (1) and table-top laser (2) pulses. Measured forward, $\mathrm{XC}(\tau)$, and backward, $\mathrm{XC}(\tau+\Delta t)$, cross-correlation signals as a function of the delay $\tau$ between FEL and table-top pulse are used to determine the balanced value, BOC $(\tau, \Delta t)$. (b) Illustration of normalized crosscorrelation and BOC functions. The BOC curve is calculated according to Eq. (1). 
radiation and NIR table-top pulses in gallium selenide (GaSe). This nonlinear material has proven to be a wellsuited medium for IR and $\mathrm{THz}$ applications [29], due to its wide transparency range $(\lambda=0.6-20 \mu \mathrm{m})$ and strong $\chi^{(2)}$-nonlinearity [30]. Both cross-correlations are performed in the same crystal, whereby the incoming focused beams have a noncollinear geometry (angle of $15^{\circ}$ ). By appropriately adjusting the angle of the crystal surface normal with respect to the input beams, we achieve efficient (type II) phase-matching in the $1 \mathrm{~mm}$ thick z-cut GaSe crystal, such that standard silicon photodiodes are sufficient to detect the SFG signal in the NIR spectral range with single micropulse resolution. Due to momentum conservation, the SFG beam is almost collinear with the NIR FO beam. Therefore, we spectrally separate the SFG signal from the FO radiation using a dichroic mirror and shortpass filters. The FEL beam is focused (first cross-correlation) and refocused (second cross-correlation) by an off-axis parabolic and spherical gold mirror with focal lengths of 300 and $150 \mathrm{~mm}$, respectively. Reference FEL power measurements using the photoelectromagnetic detector allow us to cancel the effect of FEL macropulse-to-macropulse intensity fluctuations. The data shown in the following were taken at an FEL wavelength of $10 \mu \mathrm{m}$ with $\sim 1 \mu \mathrm{J}$ micropulse energy at a repetition rate of $27.8 \mathrm{MHz}$ (or $1 \mathrm{GHz}$, respectively) and $\sim 5 \mathrm{~nJ}$ FO pulse energy. All measurements have been performed at ambient conditions.

\section{Timing characteristics}

Using the balanced optical cross-correlator, the shorttime variations (jitter) of the FEL-table-top laser pulse synchronization as well as long-term fluctuations (drift) are acquired. A typical shot (i.e., macropulse)-resolved BOC curve is shown in Fig. 2(a). The dynamic range of the implemented timing tool captures about $3 \mathrm{ps}$, whereas the
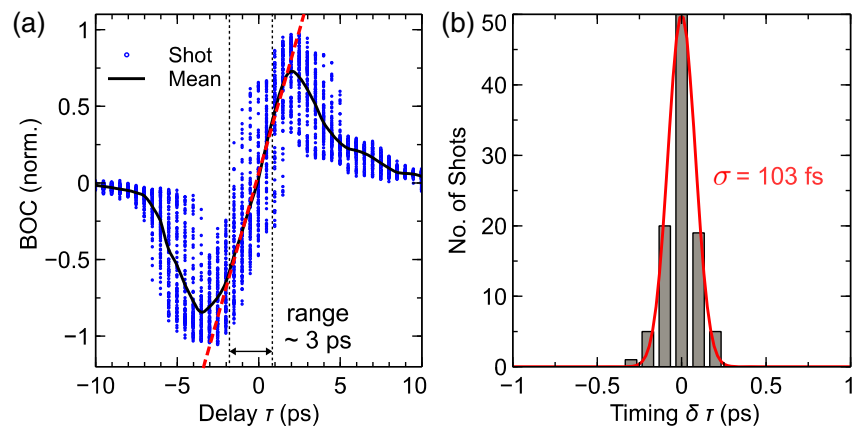

FIG. 2. Jitter determination by balanced optical crosscorrelation. (a) Measured BOC curve by varying the FEL-tabletop laser pulse delay $\tau$ for an average of 100 FEL macropulses (blue points) per delay step. The linear region (red line) of the averaged function (black line) represents the dynamic range of the tool (about $3 \mathrm{ps)}$ ). Analyzing the delay values at BOC zerocrossing, the timing statistics shown in the histogram plot in (b) is obtained. By fitting a Gaussian distribution, a jitter value of $\sigma \sim 100 \mathrm{fs}$ is extracted.
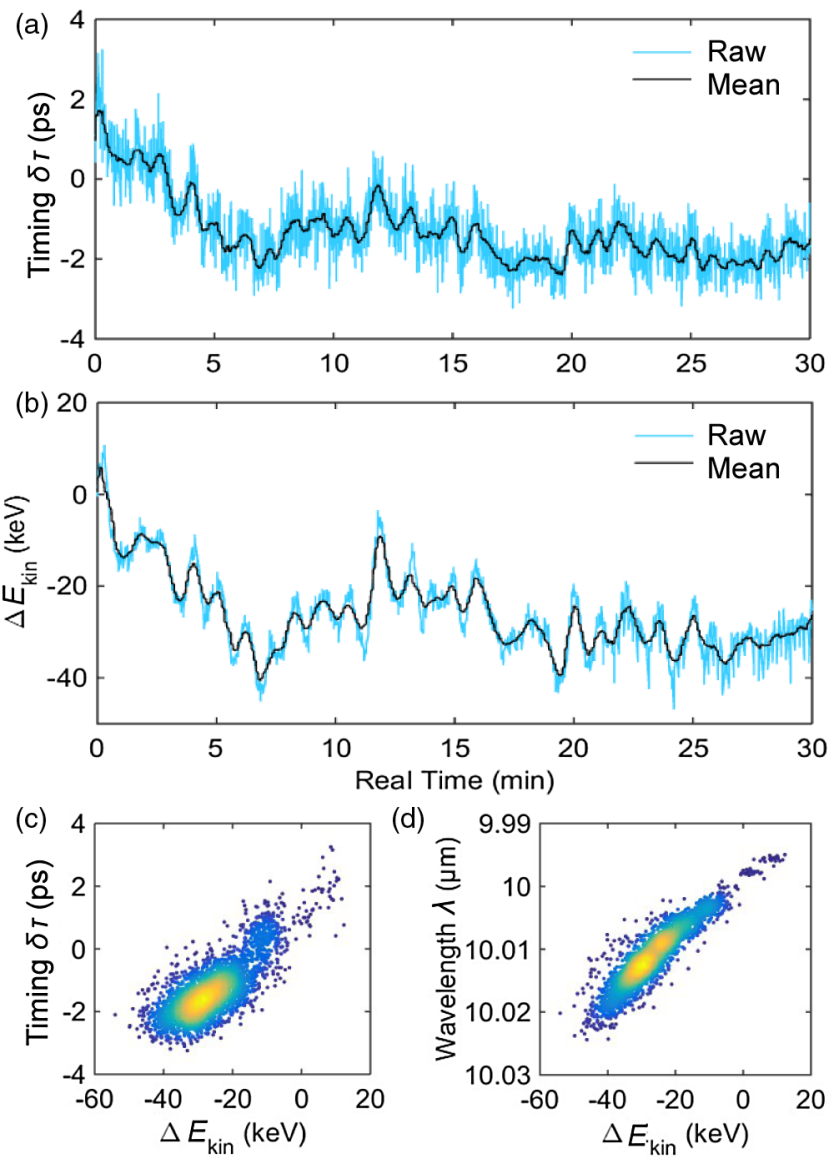

FIG. 3. Drift of FEL pulse timing and electron energy. (a) Micropulse timing fluctuations $\delta \tau$ measured by calibrated BOC (blue curve) and moving average with half-minute window (black curve) and (b) corresponding changes in kinetic energy $\Delta E_{\text {kin }}$ of electron bunches as determined by beam-position monitoring. Nominal electron energy is $E_{\text {kin }, 0}=36.5 \mathrm{MeV}$. In (c) and (d), the correlation of the raw data is shown by scatter plots with color indicating data density (blue low, yellow high). The calculated (Pearson) correlation coefficient $\rho$ is 0.78 and 0.92 between kinetic energy vs micropulse timing (c), and FEL radiation wavelength $(\mathrm{d})$, respectively.

resolution is in the fs range, solely limited by SFG intensity fluctuations and the jitter of the rf synchronization. Analyzing the BOC curve for one hundred subsequent FEL macropulses, the shift of the zero-crossing is used for jitter determination by a Gaussian fit of the normally distributed timing variations [Fig. 2(b)]. The micropulse jitter value is on the order of $\sigma \sim 100 \mathrm{fs}$ in the $27 \mathrm{MHz}$ low-repetition mode and about $\sigma=200-300 \mathrm{fs}$ in case of the $1 \mathrm{GHz}$ FEL rate. No significant dependence of the IR pulse jitter on the FEL wavelength (different undulator gap size for given electron energy) or FEL cavity length (mirror translation) has been found. It is the lowest reported jitter value compared to other IR FEL facilities [31-33].

A measurement of the timing drift is depicted in Fig. 3(a), together with a simultaneous recording of the kinetic energy changes of the accelerated electron bunches, Fig. 3(b). 
The energy variations are traced by a calibrated horizontal beam-position monitor, following a magnetic bend of the electron beam after the two linacs. The peak-to-peak timing fluctuation is about 3 ps per half-hour. As depicted in Fig. 3(c), a clear linear correlation (coefficient $\rho=0.8$ ) is found between the changes of the electron energy and the timing drift. The energy variations over time, $\Delta E_{\text {kin }} / E_{\text {kin, } 0}$, of up to $0.1 \%$ are in the same order of magnitude as the energy spread of a single electron bunch. Since the magnetic undulator field is fixed, the kinetic energy fluctuations are directly reproduced in the shift of the FEL center wavelength $[\rho=0.9$, Fig. 3(d)], in accordance with the resonance condition.

Since a table-top oscillator [23] and clock transfer system [25] of high timing stability are employed, the main contributions to the temporal fluctuations are assigned to the electron acceleration system of the FEL. Small amplitude and phase fluctuations of the rf fields in the electron gun, buncher cavity, and the two linacs can lead to variations in bunch timing and electron energy. Dispersive effects along the electron transfer beamline to the undulator can then convert kinetic energy shifts into bunch timing differences. Notably, no feedback systems are applied to the accelerator system to stabilize the electron bunch energy or arrival time. Drifts due to thermal expansion of the IR beamline are found to be of minor impact during the investigated period of time. As mentioned above, environmental phase shifts in the rf transfer from the FEL vault to the FO laser are canceled out by the actively stabilized fiber distribution system. The larger short-time fluctuations for the standard FEL repetition rate may be ascribed to the higher overall beamload of the linacs, resulting in larger thermal fluctuations of the accelerating rf fields. Considering that the synchronization relies solely on radio-frequency-based phaselocking, a high stability of the pulse timing is achieved, suitable for sub-ps resolved FEL-table-top experiments.

In order to perform ultrafast FEL pump-table-top probe studies with femtosecond precision, the calibrated balanced optical cross-correlator can be operated in parallel to the actual experiment. On a single-shot basis, the real-time deviation of the FEL pulse arrival time with respect to the delay-stage controlled table-top laser probe is monitored. Post-correction of the delay value is applied to compensate for timing drifts. In general, time-resolved spectroscopy utilizing fourth generation accelerator-based light sources requires precise timing information. Here, we characterize the FEL pulse arrival directly in a simple, all-optical way. Due to the linearity of the measured observable to absolute timing conversion, the method of BOC is superior to a single SFG cross-correlator, as it has been applied to IR FELs previously $[9,10]$.

\section{FEL PULSE PROFILE}

Using the low-jitter synchronized laser system, we are able to study the temporal profile of the FEL micropulse by
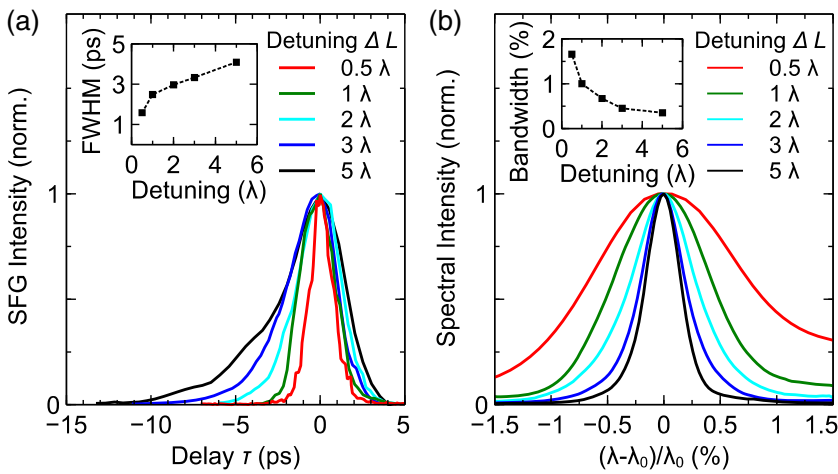

FIG. 4. FEL pulse profile dependence on cavity detuning $\Delta L$. (a) Temporal shape $I(\tau)$ of the micropulse measured by SFG cross-correlation and extracted FWHM duration (inset). (b) Spectral structure and relative bandwidth $\Delta \lambda / \lambda$ (inset); all data taken at a wavelength of $\lambda_{0}=10 \mu \mathrm{m}$.

sum-frequency cross-correlation. In contrast to optical auto-correlation measurements, cross-correlation of the FEL with a table-top laser pulse of known temporal profile allows one to determine the intensity envelope of the emitted FEL pulse. Since the reference pulse used from the FO is about one order of magnitude shorter than the investigated FEL pulse, the measured convolution trace represents in close approximation the actual FEL pulse shape. The dependence of the pulse profile on the cavity detuning $\Delta L$ is depicted in Fig. 4(a). The graphs show the micropulse structure integrated over the whole macropulse. Here, the leading edge of the micropulse is located at negative delay times. Note that, at zero detuning no steadystate lasing is established due to lethargy of the FEL, resulting in zero net gain [16].

For small detuning, $0<\Delta L \lesssim 1 \lambda$, a Gaussian-shaped pulse intensity profile $I(\tau)$ is observed. Further shortening of the FEL cavity length results in an increased pulse duration, which is on the order of a few picoseconds [see inset of Fig. 4(a)]. Additionally, a transition to an asymmetric micropulse shape with an exponential leading edge can be observed. This behavior is caused by a varying temporal overlap between the short ps optical pulses and electron bunches. On successive round-trips, the shortened cavity length shifts the free-electron gain medium to the rear edge of the IR pulse that is amplified, while the front end experiences resonator loss. These cavity-dependent pulse-profile results are in qualitative agreement with observations at other IR FEL oscillators with normal- [34] and superconducting linacs [32].

The corresponding narrowing of the FEL radiation spectrum for a stronger detuned cavity is depicted in Fig. 4(b). A relative spectral bandwidth as low as $0.3 \%$ can be realized. On basis of the macropulse integrated data, the calculated time-bandwidth product develops from 0.78 at small detuning $(\Delta L=0.5 \lambda)$ to 0.42 at large detuning $(\Delta L=5 \lambda)$. These values are about a factor of two larger than expected for a nearly transform-limited 
Gaussian-shaped pulse at small detuning. Previous work had argued that the FEL micropulses are expected to be transform-limited [21,34]. We ascribe this discrepancy to the evolution of the micropulse timing during the macropulse, i.e., an effective macropulse chirp, as detailed in the following section.

Overall, cavity detuning provides the possibility to control the duration and bandwidth of the emitted FEL radiation. On the one hand, short pulses for time-resolved experiments are available; on the other hand, narrowband radiation can be used for high-resolution nonlinear spectroscopy $[35,36]$ or selective excitation of quantum states in matter.

\section{EVOLUTION OF FEL PULSES}

\section{A. Experimental results}

Details of how the micropulse intensity profile evolves within the macropulse are revealed by analyzing the fully delay- and real-time-resolved SFG cross-correlation data. The two-dimensional plots in Figs. 5(a) and 6 show the development of the pulse profile $I(\tau)$ (vertical axis) as function of the temporal position within the macropulse (horizontal axis), i.e., the number of cavity round-trips. As apparent from the different pulse patterns shown for $\Delta L=1 \lambda-3 \lambda$, the FEL cavity detuning is found to be a highly sensitive parameter. First, we will discuss the pulse
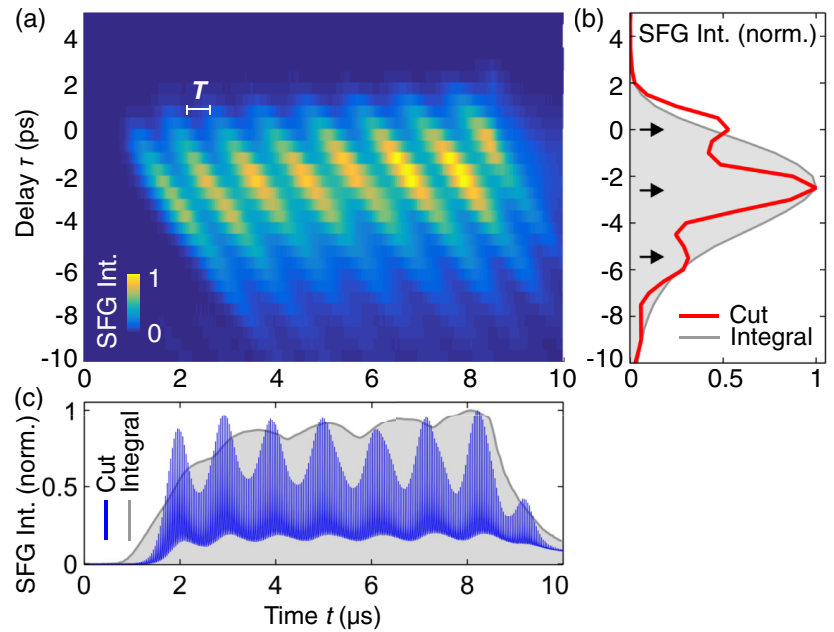

FIG. 5. Subpulse formation and limit-cycle oscillations of the FEL oscillator. Evolution of the FEL pulse shape $I(\tau)$ is obtained by SFG cross-correlation at $\Delta L=1.75 \lambda$. Cross-sections from the 2D plot (a) are along the constant time $t=8 \mu \mathrm{s}$ (b) and the constant delay $\tau=-3$ ps (c), respectively. The composition of the micropulse of equally spaced subpulses (i.e., multiple peaks) in (b) and the limit-cycle power oscillation within the macropulse (c) are directly resolved. The time- or delay-integrated intensity envelopes are plotted for comparison in (b) or (c), respectively (grey area). Temporal separation of the subpulses [marked by arrows in (b)] is 2.7 ps. The amplitude modulation of the individual micropulses is clearly visible in (c).
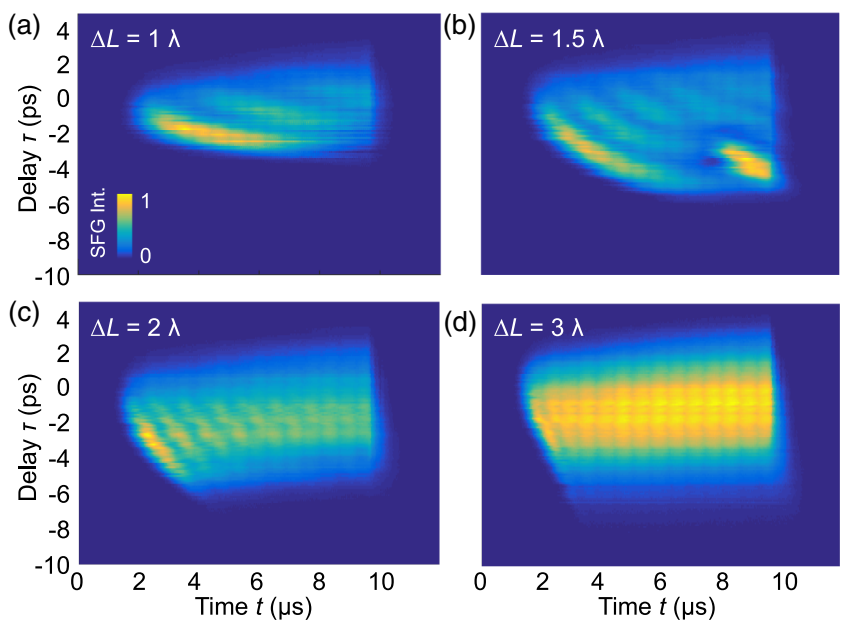

FIG. 6. Evolution of the micropulse shape $I(\tau)$ within a macropulse of the FEL oscillator at different cavity detunings. The data for $\Delta L=1 \lambda-3 \lambda$ are measured by SFG crosscorrelation (linear scale of SFG intensity). Limit-cycle oscillations of the FEL power are clearly resolved in the cases of (a)-(c).

evolution at an intermediate detuning value (Fig. 5), where pronounced power oscillations within the macropulse appear, before analyzing the dependence of these effects on the cavity detuning.

Generally, the optical output is determined by the interplay of gain (supplied by the free-electron medium) and resonator losses (described by the $Q$ factor). In Fig. 5(a), the pulse development at $\Delta L=1.75 \lambda$ is depicted. At the beginning of the macropulse, a single optical micropulse begins to develop as soon as the gain exceeds the lasing threshold. After a number of undulator passes, its peak position moves to earlier delay times, due to the detuning mechanism. On the trailing edge $(\tau>0)$, a second subpulse is initialized. With further cavity roundtrips, the pulse structure is continuously shifted along the delay axis. New subpulses arise if there is net gain, whereas the amplitude decays on the leading edge due to resonator loss. While shifting, the relative temporal distance of the subpulses is kept constant, resulting in a periodic modulation of the FEL output. A cut of the cross-correlation graph parallel to the delay axis represents the actual micropulse shape at a certain point of time within the macropulse [Fig. 5(b)], which is in contrast to the macropulse-integrated data [cf. Fig. 4(a)].

The cross-section of the SFG data along the real-time axis for a fixed delay $\tau$, see Fig. 5(c), clearly reveals regular oscillations of the emitted FEL power during the macropulse. The strength of the intensity modulation amounts to up to $50 \%$, in contrast to the delay-integrated total power oscillations, which show much weaker modulation. The osillations are a consequence of the subpulse formation. The extracted oscillation period $T$ [indicated in Fig. 5(a)] is characteristic for a certain detuning value. As will be discussed below, these modulations can be understood as a specific type of limit-cycle oscillations. 


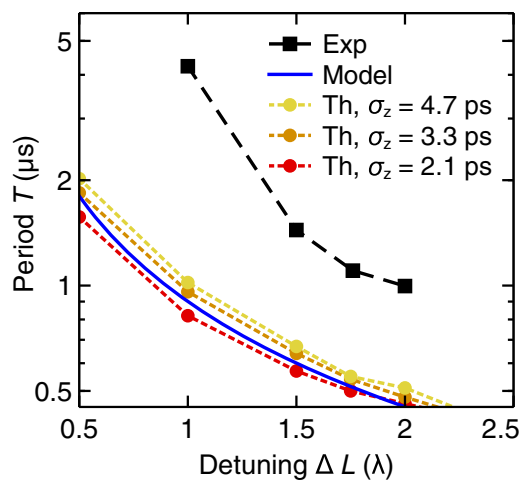

FIG. 7. Dependence of the LCO period $T$ on the cavity detuning $\Delta L$. The oscillation period is indicated in Fig. 5(a), experimental values are also extracted from the data shown in Fig. 6. The analytic model function is according to Eq. (2) and described in the text. Simulation results are obtained from 4D calculations based on Maxwell-Lorentz theory for different electron pulse durations $\sigma_{\mathrm{z}}$.

A series of two-dimensional cross-correlation measurements for different cavity detunings $\Delta L$ is shown in Fig. 6. At small detunings, the optical subpulses and LCO of the emitted power are clearly present. However, the period and strength of the intensity modulation diminish with increasing cavity detuning. This is summarized by the extracted $T$-values given in Fig. 7. For large values of $\Delta L(\gtrsim 3 \lambda)$, no significant power variations across the macropulse are observed. The distortion of the regular pattern in Fig. 6(b) might be attributed to the particular alignment of the electron beam with respect to the undulator axis, since the FEL pulse evolution is very sensitive to the position and angle of the beam entrance [16]. Measurements at other beamtimes do not show this particular irregularity, however, details of the electron beam injection into the undulator are not routinely monitored at the FEL facility. The switch-off of the $10 \mu \mathrm{s}$ long electron macrobunch determines the end of the gain for the optical macropulse. In consequence, a clear edge in the intensity profile appears at $\sim 10 \mu \mathrm{s}$ in all cases (a)-(d) of Fig. 6. Subsequent decay of the FEL intensity is governed by the cavity $Q$ factor.

Our experimental observations are in agreement with previous investigations of the optical pulse dynamics in other IR free-electron laser oscillators [5,7], however, not seen in such clarity as here due to the delay- and real-timeresolved cross-correlation measurements. The subpulse creation and power oscillations arise without external perturbation, caused by a nonlinear interaction of the short electron bunches and optical pulses. Similar to the behavior of other two-dimensional dynamical systems in mechanics [37], the self-sustained oscillation represents a limit-cycle mode. In a small-gain FEL oscillator regime without limit-cycle oscillations, the group velocity of the optical pulses $v_{\mathrm{g}}$ within the undulator is essentially determined by the gain-providing electron bunches, hence slower than the speed of light $c_{0}$. Upon saturation of the radiation intensity, $v_{\mathrm{g}}$ is getting closer to the vacuum value $c_{0}[7,21]$. Consequently, the optical pulse travels ahead of the electron bunch, leading to a reduced energy extraction and decay. Experiencing gain on the trailing edge of the main pulse, a new subpulse arises, growths and decays by the same mechanism. The repeated variation of the optical group velocity creates a train of equally-spaced subpulses, resulting finally in a regular pattern of optical power oscillations. Since larger detuning values entail lower power saturation, the oscillation depth is getting smaller until a regime with a uniform micropulse shape and constant intensity during the macropulse is settled [Fig. 6(d)].

Considering the electron motion: An increasing optical field strength leads to a stronger modulation of the particles' energy. Caused by the energy spread, the optical gain is decreasing. In a saturated, strong optical field electrons are trapped to a closed path in phase space, performing synchrotron oscillations while transversing the undulator [21]. During such an oscillation period, the particles shift back one slippage length $s=N \lambda$ in relation to the optical pulse. At the same time, the electrons' reabsorption of power from the radiation field reduces the gain to below threshold. Then, at the end of the optical pulse, electron bunches can again provide energy to the field, thus forming a new subpulse. Consequently, the LCO period of the macropulse power, Fig. 7, is generally determined by the time that is necessary for a subpulse to propagate the slippage distance $s[5]$ :

$$
T=\frac{s}{\Delta L} \frac{L_{0}}{c_{0}} .
$$

Our measurements agree with the trend of decreasing oscillation period for shorter FEL cavity length. The comparison with a more refined formula, considering the shorter slippage distance within a synchrotron period instead of the full slippage length [38], does not improve the agreement. Reasons for the quantitative deviation of our data from the model formula are discussed below.

Additionally, intensity autocorrelation measurements of the FEL micropulse as a function of cavity detuning have been performed to further illustrate the IR pulse behavior. Utilizing second-harmonic generation (SHG) in cadmium telluride [39] in a crossed-beam reflection geometry [6], the background-free correlation function is recorded by a $\mathrm{HgCdTe}$ detector. The delay- and time-resolved autocorrelation results at an FEL wavelength of $12 \mu \mathrm{m}$ and a micropulse rate of $1 \mathrm{GHz}$ are shown in Fig. 8. Since the detector response is slower than the repetition period, individual micropulses cannot be distinguished along the time axis. Near zero detuning $(\Delta L=0.5 \lambda)$, a single pulse with a duration of $420 \mathrm{fs}$ (FWHM) is generated, assuming a Gaussian intensity envelope [Fig. 8(a)]. This corresponds to the length of about 10 cycles of the optical field. By shortening of the FEL cavity, Figs. 8(b) and 8(c), side peaks emerge symmetrically due to the formation of subpulses 


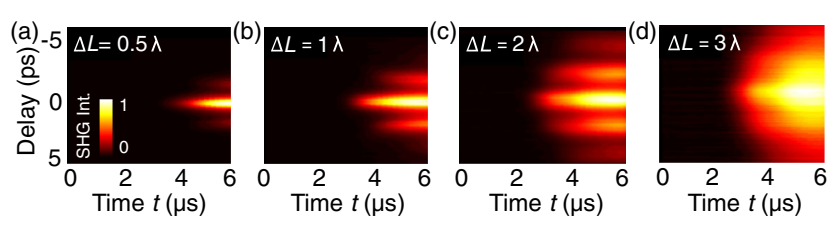

FIG. 8. Subpulse structure as seen by SHG auto-correlation measurements at different detunings $\Delta L$. In the plots (a)-(d), only the beginning of the macropulse is shown, while the delay refers to the temporal overlap of the split micropulse. For shorter cavity lengths, more subpulses are formed until their overlap results in a single-peaked, broad micropulse.

as mentioned above. The build-up of these pulses occurs as soon as the main pulse approaches saturation. For large detunings, a single-peaked, temporally long micropulse is developed [Fig. 8(d)]. This can be seen as an overlap of many optical subpulses with narrow temporal spacing. Thus, the autocorrelation results are consistent with the SFG measurements, however, they provide less information about the subpulse development due to the inherent symmetry.

\section{B. Simulations}

The dynamics of electron motion and optical waves within a small-gain FEL oscillator are accurately described by Maxwell-Lorentz theory [16]. Here, we have performed four-dimensional simulations of the FHI FEL, including longitudinal $(z)$ and transversal $(x, y)$ evolution of the optical field as function of time $(t)$. These are used to trace the build-up of the micropulse and the development of the pulse profile during subsequent roundtrips in the shortened oscillator cavity. For the rf linac-powered electron microbunches, Gaussian-shaped current pulses of finite length $\sigma_{\mathrm{z}}$ are assumed. Diffraction of the optical modes within the cavity has been taken into account, as well as hole-outcoupling of the IR radiation [19]. The parameters used for the simulations are summarized in Table I in the Appendix. The set of millions of coupled nonlinear differential equations is solved numerically and self-consistently using parallelized computation.

Theoretical results for the optical intensity $I(z, t)=$ $|a(z, t)|^{2}$ of the FEL output integrated over $(x, y)$ are shown in the 2D plots of Fig. 9 at different cavity detunings. Since the slippage distance is comparable to the electron bunch length $s / \sigma_{\mathrm{z}} \sim 1$, pronounced short-pulse effects are expected to be observed. Indeed, the formation of subpulses at small cavity detunings is clearly reproduced [Fig. 9(a)]. About $1 \mu \mathrm{s}$ after start of the electron macrobunch, i.e., $\sim 30$ resonator roundtrips, the radiation power grows considerably. The emerging optical micropulse is shifted to negative delay times due to the detuned cavity length. Subsequent intensity decay is determined by the quality factor. At the trailing edge, a new subpulse arises and undergoes the same process. For increasing detuning values [Figs. 9(b) and 9(c)], more subpulse trains are generated in shorter temporal intervals. At large values of $\Delta L$, a single-peaked micropulse shape is formed at the end of the electron macrobunch [Fig. 9(d)]. The calculated peak power of the final optical pulse $I(z)$ is decreasing for
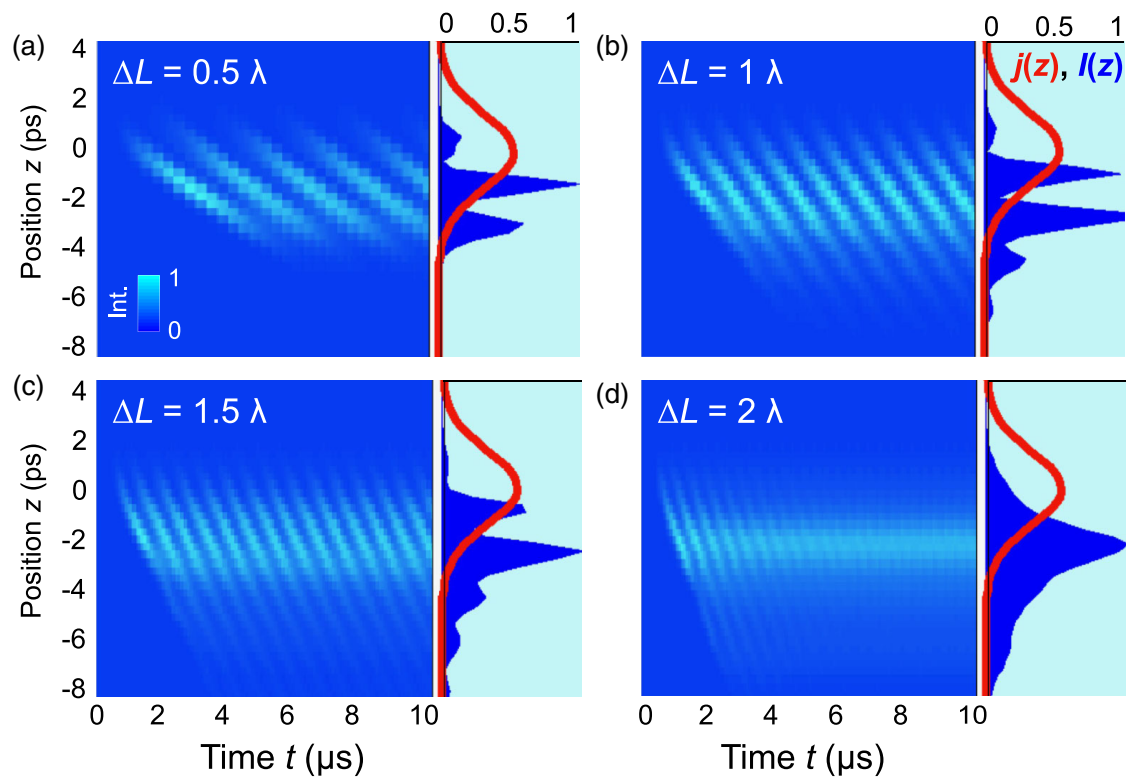

FIG. 9. Simulations of the optical pulse structure based on 4D Maxwell-Lorentz theory for different cavity detunings $\Delta L$. The twodimensional plots (a)-(d) show the evolution of the normalized FEL micropulse intensity shape $I(z)$ as function of the time $t$ within the macropulse. Line graphs on the right present the optical pulse profile (blue) and electron bunch density $j(z)$ (red line) at the end of macropulse and undulator. The train of equally spaced subpulses is clearly reproduced. At the larger detuning value, a single-peaked asymmetric micropulse emerges. The ratio of the optical peak intensity evolves as 15:5:3:1 from $\Delta L=0.5 \lambda$ to $2 \lambda$. 
smaller cavity lengths due to the diminishing overlap with the free-electron gain medium $j(z)$.

The LCO period $T$ extracted from the simulations, i.e., the distance between the intensity peaks along the time axis $t$, is shown in Fig. 7, together with the measurement data and the simple model function. The agreement between our 4D Maxwell-Lorentz simulations and the model function is excellent, considering its simplicity. The studied influence of the electron pulse duration $\sigma_{\mathrm{z}}$ on the LCO period shows that longer current pulses result in slightly increased $T$-values, however, the functional detuning dependence is not affected. It is apparent that experimental and theoretical values disagree by a factor $\gtrsim 2$. One reason is the uncertainty of the actual cavity detuning in the measurements $(\sim 0.5 \lambda)$, since the exact mirror position for zero detuning is not implemented. The position of the cavity mirror for zero detuning has been determined manually as being the case when the FEL power drops down to absolute zero. Variations of the cavity length during a cross-correlation measurement $(\sim 10 \mathrm{~min})$ are negligible. Another point is the accurate alignment of the electron injection with respect to the undulator $z$-axis: Position and angle of the electron beam are initially adjusted for maximum IR power extraction and are not monitored. Further work is intended to study the influence of the electron beam alignment on the optical pulse shape and spectrum, both experimentally and by simulations, potentially resolving the disagreement.

In general, the LCO period is determined by the interplay of optical field strength, electron pulse profile, and cavity detuning. Due to advances in modeling and computation power nowadays, the full space- and timedependent simulations shown in Fig. 9 allow a detailed picture of the FEL lasing, which is a considerable improvement compared to the first prediction of limitcycle oscillations [20]. Although the generation of a self-modulated pulse shape is a peculiar property of the nonlinear electron-light interaction by itself, LCOs of the FEL power might be disadvantageous in timeresolved spectroscopy. Usable single-peaked FEL pulses are obtained at larger detunings $(\Delta L \gtrsim 3 \lambda)$, where also a small spectral bandwidth $(<0.5 \%)$ is present, suitable for selective excitation. But, besides a reduction of the extracted IR power from the FEL, the time resolution is diminished in the so-called stable-focus regime due to an increased micropulse duration ( $>3 \mathrm{ps}$ ).

Finally, we note that sub-ps micropulses consisting of a single subpulse are available for time-resolved experiments as well, if the FEL cavity detuning $\Delta L$ is close to zero. However, operation of the FEL oscillator in that regime results in an unstable lasing output, both in terms of pulse arrival time and intensity [16]. This limits the applicability to sub-ps two-color pump-probe studies. Nevertheless, the precise timing of the IR FEL radiation with respect to the table-top laser will be essential for other applications like VIS-IR sum-frequency generation spectroscopy with interface sensitivity. Hence, the study of the FEL pulse profile presented here complements the characterization of the synchronized laser setup for planned spectroscopic applications.

\section{CONCLUSION}

We have established and demonstrated a method to determine the pulse arrival time at a long-wavelength FEL with fs-accuracy on a single-shot basis. Utilizing balanced optical cross-correlation with synchronized ultrashort table-top laser pulses, the FHI FEL timing jitter is found to be as low as $100 \mathrm{fs}$, at least one order of magnitude smaller than the micropulse duration. In comparison to previous IR FEL facilities, it is the lowest reported temporal jitter. Also, we have revealed a distinct relationship between the FEL pulse arrival time and the mean kinetic energy of the electrons. The temporal drift in the ps range per hour is found to be directly correlated with energy fluctuations of the driving electron bunches. Thus, our observations indicate that active stabilization of the amplitudes and phases of the accelerator rf fields by an automized feedback will reduce drifts of both the electron-bunch arrival time and its mean kinetic energy significantly. In addition to a decrease of the FEL pulse drift to the sub-ps level, spectral fluctuations of the FEL output might be minimized as well. However, further investigations on the exact cause of the optical pulse drift are necessary for an efficient feedback system. Besides the technical improvements, the concept of balanced arrival time detection is a robust method applicable to FEL pulses, superior to single cross-correlation measurements. This enables post-correction of the FEL pulse timing in pump-probe spectroscopy, circumventing the requirement for a strict synchronization at accelerated-based light sources. The BOC concept is not limited to sum-frequency generation and can be employed to other spectral regions of FEL and table-top laser radiation as well, provided that a cross-correlation function can be measured.

Using our synchronization scheme, we have presented a comprehensive experimental and theoretical study of subpulse formation and dynamics in a low-gain FEL oscillator operating in the limit-cycle regime. Two-dimensional cross-correlation measurements provide direct access to the evolution of the optical pulse structure, showing the growth of a train of subpulses within a well-defined period, subsequent temporal shifting and decay. Time-dependent four-dimensional FEL simulations based on self-consistent solutions of Maxwell-Lorentz theory confirm the generation process, which occurs at moderate detuning values if the electron bunch length is on the order of the slippage length. The resulting limit-cycle oscillations of the FEL intensity on a microsecond timescale are revealed with high clarity, reinforcing the established theoretical understanding with experimental data that go beyond previous observations. Monitoring of the actual two-dimensional intensity profile may facilitate the optimization of the lasing 
process, supported by detailed simulations, in order to increase FEL power and pulse timing performance.

\section{ACKNOWLEDGMENTS}

We thank M. Kaiser and S. Hunziker from Paul Scherrer Institute $(\mathrm{CH})$ for helpful discussions and valuable support regarding the rf synchronization system.

\section{APPENDIX: SIMULATION PARAMETERS}

The parameters of the FHI FEL used in the 4D simulations of the optical pulse structure are summarized in Table I.

TABLE I. FHI FEL parameters of electron bunches, undulator, and cavity used in the simulations.

\begin{tabular}{lc}
\hline \hline Parameter & Value \\
\hline Electron energy $E_{\text {kin }}$ & $36.5 \mathrm{MeV}$ \\
Energy spread & $<50 \mathrm{keV}$ \\
Bunch charge & $200 \mathrm{pC}$ \\
Bunch length $\sigma_{\mathrm{z}}$ & $3 \mathrm{ps}$ \\
Micropulse repetition rate $f_{\text {rep }}$ & $27.8 \mathrm{MHz}$ \\
Macropulse length & $10 \mu \mathrm{s}$ \\
Normalized rms transverse emittance & $\pi 20 \mathrm{~mm} \mathrm{mrad}$ \\
Lorentz factor $\gamma$ & 72.4 \\
Undulator length $L_{\mathrm{U}}$ & $2.0 \mathrm{~m}$ \\
Undulator periods $N$ & 50 \\
Undulator wavelength $\lambda_{\mathrm{U}}$ & $40.0 \mathrm{~mm}$ \\
Undulator gap $d$ & $19.5 \mathrm{~mm}$ \\
Magnetic field $B_{0}$ & $0.34 \mathrm{~T}$ \\
Undulator parameter $K$ & 1.27 \\
Optical wavelength $\lambda$ & $10 \mu \mathrm{m}$ \\
Cavity length $L_{0}$ & $5.4 \mathrm{~m}$ \\
Out-coupling hole diameter & $2.5 \mathrm{~mm}$ \\
\hline \hline
\end{tabular}

[1] M. Mittendorff, F. Wendler, E. Malic, A. Knorr, M. Orlita, M. Potemski, C. Berger, W. A. de Heer, H. Schneider, M. Helm, and S. Winnerl, Carrier dynamics in Landauquantized graphene featuring strong Auger scattering, Nat. Phys. 11, 75 (2015).

[2] H. N. Chapman et al., Femtosecond X-ray protein nanocrystallography, Nature (London) 470, 73 (2011).

[3] T. Wang et al., Femtosecond Single-Shot Imaging of Nanoscale Ferromagnetic Order in Co/Pd Multilayers Using Resonant X-Ray Holography, Phys. Rev. Lett. 108, 267403 (2012).

[4] B. W. J. McNeil and N. R. Thompson, X-ray free-electron lasers, Nat. Photonics 4, 814 (2010).

[5] D. A. Jaroszynski, R. J. Bakker, A. F. G. van der Meer, D. Oepts, and P. W. van Amersfoort, Experimental Observation of Limit-Cycle Oscillations in a Short-Pulse FreeElectron Laser, Phys. Rev. Lett. 70, 3412 (1993).

[6] W. Schöllkopf, S. Gewinner, H. Junkes, A. Paarmann, G. v. Helden, H. P. Bluem, and A. M. M. Todd, The new IR and
THz FEL facility at the Fritz Haber Institute in Berlin, Proc. SPIE 9512, 95121L (2015).

[7] G. M. H. Knippels, R. F. X. A. M. Mols, A. F. G. van der Meer, D. Oepts, and P. W. van Amersfoort, Intense FarInfrared Free-Electron Laser Pulses with a Length of Six Optical Cycles, Phys. Rev. Lett. 75, 1755 (1995).

[8] A. L. Cavalieri et al., Clocking Femtosecond X Rays, Phys. Rev. Lett. 94, 114801 (2005).

[9] A. Peremans, P. Guyot-Sionnest, A. Tadjeddine, F. Glotin, J.-M. Ortega, and R. Prazeres, Sum-frequency generation at surfaces using an infrared FEL synchronized with a YAG laser, Nucl. Instrum. Methods Phys. Res., Sect. A 331, ABS28 (1993).

[10] E. R. Eliel, E. W. M. van der Ham, Q. H. F. Vrehen, G. W. 't Hooft, M. Barmentlo, J. M. Auerhammer, A. F. G. van der Meer, and P. W. van Amersfoort, Studies of interfacial regions by sum-frequency generation with a free-electron laser, Appl. Phys. A 60, 113 (1995).

[11] C. Gahl, A. Azima, M. Beye, M. Deppe, K. Döbrich, U. Hasslinger, F. Hennies, A. Melnikov, M. Nagasono, A. Pietzsch, M. Wolf, W. Wurth, and A. Föhlisch, A femtosecond X-ray/optical cross-correlator, Nat. Photonics 2, 165 (2008).

[12] M. Harmand, R. Coffee, M. R. Bionta, M. Chollet, D. French, D. Zhu, D. M. Fritz, H. T. Lemke, N. Medvedev, B. Ziaja, S. Toleikis, and M. Cammarata, Achieving fewfemtosecond time-sorting at hard X-ray free-electron lasers, Nat. Photonics 7, 215 (2013).

[13] T. R. Schibli, J. Kim, O. Kuzucu, J. T. Gopinath, S. N. Tandon, G. S. Petrich, L. A. Kolodziejski, J. G. Fujimoto, E. P. Ippen, and F. X. Kärtner, Attosecond active synchronization of passively mode-locked lasers by balanced cross correlation, Opt. Lett. 28, 947 (2003).

[14] S. Schulz, I. Grguras, C. Behrens, H. Bromberger, J. T. Costello, M. K. Czwalinna, M. Felber, M. C. Hoffmann, M. Ilchen, H. Y. Liu, T. Mazza, M. Meyer, S. Pfeiffer, P. Predki, S. Schefer, C. Schmidt, U. Wegner, H. Schlarb, and A. L. Cavalieri, Femtosecond all-optical synchronization of an X-ray free-electron laser, Nat. Commun. 6, 5938 (2015).

[15] S. Kovalev, B. Green, T. Golz, S. Mährlein, N. Stojanovic, A. S. Fisher, T. Kampfrath, and M. Gensch, Probing ultrafast processes with high dynamic range at 4 th-generation light sources: Arrival time and intensity binning at unprecedented repetition rates, Struct. Dyn. 4, 024301 (2017).

[16] W. B. Colson, in Free Electron Lasers, Laser Handbook, edited by W. B. Colson, C. Pellegrini, and A. Renieri (North Holland, Amsterdam, 1990), Vol. 6, p. 115.

[17] C. Pellegrini, A. Marinelli, and S. Reiche, The physics of x-ray free-electron lasers, Rev. Mod. Phys. 88, 015006 (2016).

[18] D. A. Jaroszynski, R. J. Bakker, D. Oepts, A. F. G. van der Meer, and P. W. van Amersfoort, Limit cycle behaviour in FELIX, Nucl. Instrum. Methods Phys. Res., Sect. A 331, 52 (1993).

[19] J. Blau, K. Cohn, and W. B. Colson, Four-dimensional models of FEL amplifiers and oscillators, Proc. FEL2015, Daejeon, Korea (JACoW, Geneva, 2015), p. 607, https:// doi.org/10.18429/JACoW-FEL2015-WEP008.

[20] W. B. Colson, in Free-Electron Generators of Coherent Radiation, Physics of Quantum Electronics, edited by 
S. F. Jacobs, G. T. Moore, H. S. Pilloff, M. Sargent III, M. O. Scully, and R. Spitzer (Addison-Wesley, Reading, MA, 1982), Vol. 8, p. 457.

[21] R. J. Bakker, D. A. Jaroszynski, A. F. G. van der Meer, D. Oepts, and P. W. van Amersfoort, Short-pulse effects in a free-electron laser, IEEE J. Quantum Electron. 30, 1635 (1994).

[22] H. C. Schewe et al., (to be published).

[23] Onefive GmbH/NKT Photonics, Femtosecond Laser, Model: Origami $10 \mathrm{HP}$, https://www.nktphotonics.com/ wp-content/uploads/sites/3/2017/09/origami-hp-datasheet .pdf?1522182211.

[24] Instrumentation Technologies, d.d., Clock Transfer System, Model: Libera Sync 3, https://www.i-tech.si/file/ download/276_ae7a4f9baebe.

[25] S. Zorzut, M. Cargnelutti, and S. Hunziker, Influence of environment changes on libera sync 3 long-term stability, Proc. FEL2015, Daejeon, Korea (JACoW, Geneva, 2015), 126, https://doi.org/10.18429/JACoW-FEL2015-MOP043.

[26] T. Schietinger et al., Commissioning experience and beam physics measurements at the SwissFEL Injector Test Facility, Phys. Rev. Accel. Beams 19, 100702 (2016).

[27] MITEQ, Inc., Fiber Optic Link, Model: LBL-50K4P5G, https://nardamiteq.com/docs/MITEQ-LBL-50K4P5G.PDF.

[28] VIGO System S. A., IR Photoelectromagnetic Detector, Model: PEM-10.6, https://www.vigo.com.pl/pub/File/ PRODUKTY/IR/PEM/PEM.pdf.

[29] O. Schubert, M. Hohenleutner, F. Langer, B. Urbanek, C. Lange, U. Huttner, D. Golde, T. Meier, M. Kira, S. W. Koch, and R. Huber, Sub-cycle control of terahertz highharmonic generation by dynamical Bloch oscillations, Nat. Photonics 8, 119 (2014).

[30] V. G. Dmitriev, G. G. Gurzadyan, and D. N. Nikogosyan, Handbook of Nonlinear Optical Crystals (Springer, Berlin, 1999).

[31] G. M. H. Knippels, M. J. van de Pol, H. P. M. Pellemans, P. C. M. Planken, and A. F. G. van der Meer, Two-color facility based on a broadly tunable infrared free-electron laser and a subpicosecond-synchronized 10-fs-Ti:sapphire laser, Opt. Lett. 23, 1754 (1998).

[32] J. Bhattacharyya, M. Wagner, S. Zybell, S. Winnerl, D. Stehr, M. Helm, and H. Schneider, Simultaneous time and wavelength resolved spectroscopy under two-colour near infrared and terahertz excitation, Rev. Sci. Instrum. 82, 103107 (2011).

[33] X. Wang, T. Nakajima, H. Zen, T. Kii, and H. Ohgaki, Single-shot spectra of temporally selected micropulses from a mid-infrared free-electron laser by upconversion, Opt. Lett. 37, 5148 (2012).

[34] G. M. H. Knippels, X. Yan, A. M. MacLeod, W. A. Gillespie, M. Yasumoto, D. Oepts, and A. F. G. van der Meer, Generation and Complete Electric-Field Characterization of Intense Ultrashort Tunable Far-Infrared Laser Pulses, Phys. Rev. Lett. 83, 1578 (1999).

[35] I. Razdolski, Y. Chen, A. J. Giles, S. Gewinner, W. Schöllkopf, M. Hong, M. Wolf, V. Giannini, J. D. Caldwell, S. A. Maier, and A. Paarmann, Resonant enhancement of second-harmonic generation in the mid-infrared using localized surface phonon polaritons in subdiffractional nanostructures, Nano Lett. 16, 6954 (2016).

[36] A. Paarmann, I. Razdolski, A. Melnikov, S. Gewinner, W. Schöllkopf, and M. Wolf, Second harmonic generation spectroscopy in the Reststrahl band of $\mathrm{SiC}$ using an infrared free-electron laser, Appl. Phys. Lett. 107, 081101 (2015).

[37] W. M. Mansour, Quenching of limit cycles of a Van der Pol oscillator, J. Sound Vib. 25, 395 (1972).

[38] S. J. Hahn and J. K. Lee, Nonlinear short-pulse propagation in a free-electron laser, Phys. Rev. E 48, 2162 (1993).

[39] J. Xu, G. Knippels, D. Oepts, and A. van der Meer, A farinfrared broadband $(8.5-37 \mu \mathrm{m})$ autocorrelator with subpicosecond time resolution based on cadmium telluride, Opt. Commun. 197, 379 (2001). 